\title{
ESTABILIDADE DA MICROESTRUTURA DE FERROS FUNDIDOS CINZENTOS APLICADOS A ELEVADAS TEMPERATURAS*
}

\author{
Mariana Peixoto ${ }^{1}$ \\ Guilherme Ourique Verran ${ }^{2}$ \\ Wilson Luiz Guesser ${ }^{3}$
}

\begin{abstract}
Resumo
Muitos componentes de ferro fundido ficam expostos a altas temperaturas quando em serviço, podendo ocorrer a decomposição da perlita através da difusão do carbono da cementita para as lamelas de grafita, resultando em alteração microestrutural e degradação das propriedades mecânicas. A influência que a temperatura e o tempo de exposição têm sobre a microestrutura de um ferro fundido cinzento foi avaliado em amostras de quatro ferros fundidos de mesma base, com diferentes percentuais de cromo e molibdênio. Ensaios de banho de sal a uma temperatura de $500^{\circ} \mathrm{C}$ foram realizados em corpos de prova com diâmetro de $10 \mathrm{~mm}$. Os ensaios foram realizados com tempos de exposição a esta temperatura, após homogeneização térmica do material, de 0, 1, 2 e 4 horas. As análises metalográficas evidenciam a ocorrência da decomposição da perlita. Adicionalmente foram realizados ensaios de microdureza, a qual se mostrou sensível às alterações microestruturais geradas pela temperatura elevada. O ferro fundido com maior percentual de cromo e molibdênio apresentou melhor estabilidade da microestrutura para as condições ensaiadas.
\end{abstract}

Palavras-chave: Ferro fundido cinzento; Estabilidade microestrutural; Temperatura elevada.

\section{MICROSTRUCTURAL STABILITY OF GRAY CAST IRONS APPLIED TO HIGH TEMPERATURES}

\section{Abstract}

Many components of cast iron used in high temperatures applications can present decomposition of pearlite by diffusion of carbon from cementite to graphite, resulting in microstructural change and degradation of mechanical properties. It was evaluated the influence of temperature and time of exposure have on the microstructure of a gray cast iron, using four of the same cast iron base with different percentages of chromium and molybdenum. Tests at $500^{\circ} \mathrm{C}$ temperature were carried out on specimens with $10 \mathrm{~mm}$ diameter. The tests were performed with exposure time at this temperature, after thermal homogenization of the material, during $0,1,2$ and 4 hours. The metallographic analysis indicated the occurrence of decomposition of pearlite. Additionally microhardness tests were conducted, which was sensitive to microstructural changes due to high temperature. Cast iron with a higher percentage of $\mathrm{Cr}$ and Mo showed better stability of the microstructure in the tested conditions.

Keywords: Gray cast iron; Microstructural stability; High temperature.

1 Engenheira mecânica, doutoranda do Programa de Pós-graduação em Ciência e Engenharia de Materiais (PGCEM), Universidade do Estado de Santa Catarina (UDESC), Joinville, Santa Catarina, Brasil.

2 Dr. Eng. Professor do Departamento de Engenharia Mecânica (DEM) e do PGCEM, Universidade do Estado de Santa Catarina (UDESC), Joinville, Santa Catarina, Brasil.

3 Dr. Eng. Professor do PGCEM, UDESC, Diretor da Tupy S.A., Joinville, Santa Catarina, Brasil. 


\section{INTRODUÇÃO}

Inúmeras peças de ferro fundido, tais como blocos e cabeçotes de motor, são submetidas a elevadas temperaturas de trabalho. A seleção de ferros fundidos para aplicações em temperatura elevada é uma tarefa de especial importância [1, 2].

De acordo com Guesser [1], ferros fundidos aplicados em altas temperaturas devem possuir algumas características importantes, como: estabilidade microestrutural, baixa variação de propriedades mecânicas, resistência à oxidação, baixa tendência ao crescimento e alteração da microestrutura, e, resistência à fadiga térmica. A figura 1 mostra estas relações.

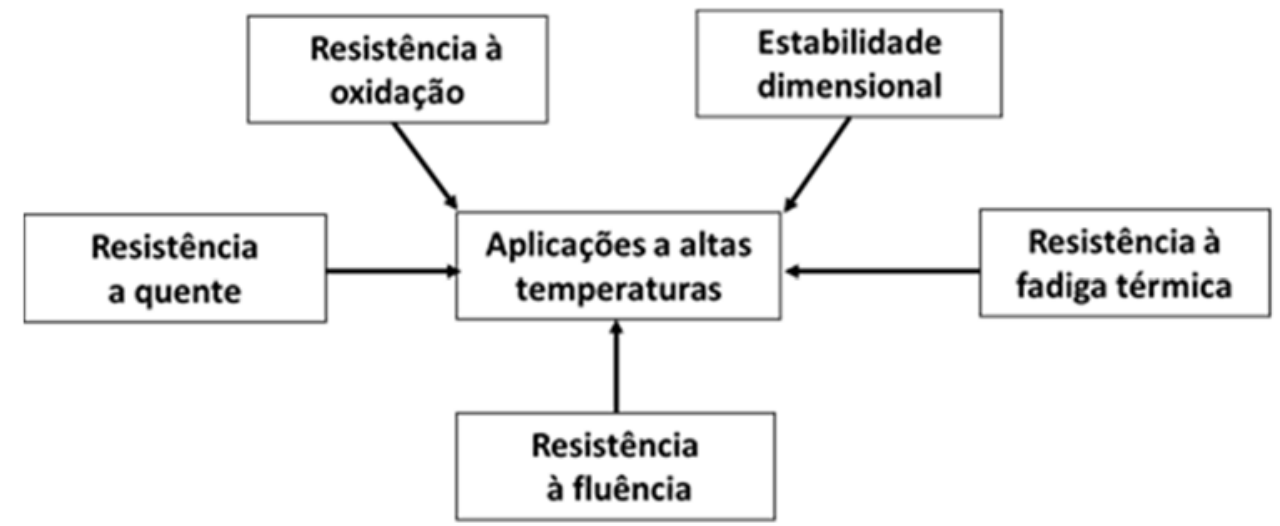

Figura 1. Fatores importantes para aplicações de ferros fundidos a elevadas temperaturas [1].

Os ferros fundidos cinzentos são ditos estruturalmente estáveis até temperaturas de $350^{\circ} \mathrm{C}$. A grande área interfacial ferrita/cementita na estrutura lamelar da perlita indica que uma estrutura com uma área interfacial menor, tal como cementita esférica numa matriz ferrítica, seria muito mais estável [3]. Devido a isto, Palmer [4] recomenda que acima de $350^{\circ} \mathrm{C}$ seja utilizado ferros fundidos ligados, pois a partir deste ponto observa-se o inicio do processo de esferoidização da perlita durante o tempo de exposição a elevada temperatura. Além disto, a cementita, que já é termodinamicamente instável, começa a decompor-se em ferrita e grafita $[5,6]$. 0 carbono livre migra então, por difusão, para a grafita já existente na matriz, aumentando o seu percentual na microestrutura. $O$ aumento da temperatura de trabalho do material acelera a velocidade de difusão, o que acaba por intensificar o processo de decomposição da cementita [7].

A estabilidade microestrutural de um ferro fundido é de fundamental importância para uso em elevadas temperaturas, a qual pode ser também observada pela diminuição no limite de resistência, diminuição da dureza e por variações dimensionais [8, 9].

Vários autores $[6,10]$ demonstraram o decréscimo da dureza em ferros fundidos quando expostos a elevadas temperaturas. Gilbert e White [6] notaram uma queda na dureza em ensaios realizados a $500^{\circ} \mathrm{C}$ e $650^{\circ} \mathrm{C}$, a qual foi acompanhada de alteração microestrutural. A figura 2 mostra o comportamento da dureza de diferentes ferros fundidos após aquecimento à $650^{\mathrm{a}} \mathrm{C}$.

A análise da estabilidade microestrutural de ferros fundidos cinzentos perlíticos com diferentes teores de cromo e molibdênio foi realizada através de ensaios em banho de sal a $500^{\circ} \mathrm{C}$, com tempos de exposição de $0,1,2$ e 4 horas. Análises metalográficas foram utilizadas para observar o comportamento dos materiais a elevada temperatura em função dos tempos de exposição, e os fenômenos 
envolvidos nas variações das propriedades mecânicas dos mesmos. Adicionalmente, a pesquisa contempla a avaliação da microdureza em função do tempo de exposição a elevadas temperaturas.

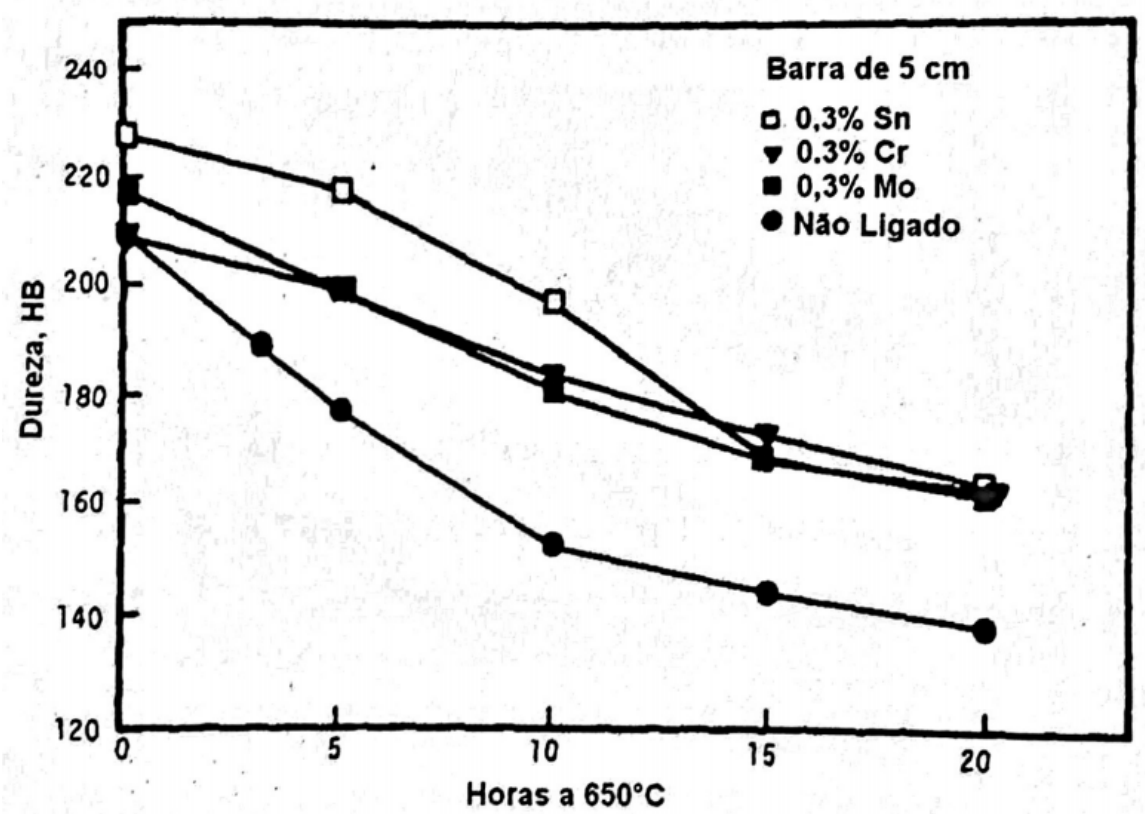

Figura 2. Influência do estanho, cromo e molibdênio na dureza de ferros fundidos cinzentos após aquecimento à $650^{\mathrm{a}} \mathrm{C}[11]$.

O ferro fundido cinzento perlítico de baixa liga apresenta microestrutura composta por grafita em forma de veios, constituindo um esqueleto contínuo nas células eutéticas, além de uma matriz perlítica e carbonetos [1, 12]. A formação de carbonetos está relacionada com o teor de elementos de liga adicionados ao material.

O papel dos elementos de liga é extrema importância para aplicações a elevadas temperaturas. Normalmente, os maiores benefícios são alcançados quando vários elementos de liga são usados em combinação. Elementos de liga como $\mathrm{Cr}$ e Mo inibem a velocidade da decomposição da perlita em ferros fundidos cinzentos expostos a elevadas temperaturas [13]. O cromo reduz o teor de carbono livre e diminui o espaçamento da perlita, o que produz um efeito acentuado na diminuição da decomposição microestrutural, na resistência ao crescimento e à oxidação. No entanto, tem um efeito menor sobre a resistência mecânica e à fluência [14]. $O$ molibdênio, por sua vez age refinando a perlita, sendo um dos elementos mais atuantes para o aumento da resistência mecânica a quente, além de possuir menor tendência ao coquilhamento do que outros elementos estabilizadores de carbonetos [15].

\section{MATERIAIS E MÉTODOS}

Para o presente estudo foram utilizados quatro ferros fundidos cinzentos perlíticos ligados, possuindo diferentes percentuais de cromo e molibdênio, como mostrado na tabela 2. Ainda elementos de liga como cobre e estanho estão presentes nas ligas.

Os corpos de prova foram fornecidos pela empresa TUPY S.A., os quais foram obtidos a partir de um pino bruto de fundição. Cada corpo de prova possui $30 \mathrm{~mm}$ de 
diâmetro e $15 \mathrm{~mm}$ de altura (com material proveniente da mesma corrida) para a realização dos ensaios de estabilidade da microestrutura a elevada temperatura.

Tabela 2. Composição química das ligas

\begin{tabular}{ccc}
\hline \multirow{2}{*}{ Liga } & \multicolumn{2}{c}{ \% em peso } \\
& Cromo (Cr) & Molibdênio (Mo) \\
\hline C1 & 0,103 & 0,002 \\
C2 & 0,284 & 0,002 \\
C3 & 0,106 & 0,294 \\
C4 & 0,287 & 0,296 \\
\hline
\end{tabular}

Para o estudo da estabilidade térmica da microestrutura foram realizados ensaios em banhos de sais no Centro de Tratamentos Térmicos da UNISOCIESC. Para os ensaios foram utilizados sais específicos para a faixa de temperatura avaliada, o que garantem a integridade microestrutural do material, sem influenciar nos resultados. O banho foi realizado a uma temperatura de $500^{\circ} \mathrm{C}$, onde os corpos de prova ficaram no banho por diferentes intervalos de tempo, os quais foram de 0, 1, 2 e 4 horas (com um tempo de homogeneização inicial de $5 \mathrm{~min}$ ).

A determinação do percentual de grafita presente na microestrutura dos corpos de prova ensaiados foi realizada através de análise metalográfica, que utilizou 40 imagens aumentadas em 100x, para cada amostra. $O$ valor percentual resultante de cada amostra considerou a média destes resultados obtidos a cada temperatura.

Todos os corpos de prova tiveram também sua microdureza determinada. Cada amostra teve 10 medições aleatórias, que foram realizadas em laboratórios da empresa TUPY S.A. em um microdurômetro da marca Shimadzu, modelo DUH W211 Series Micro Hardness Tester, com penetrador em forma de pirâmide triangular com ângulo de $115^{\circ}$, com carga de $0,2 \mathrm{mN}$.

\section{RESULTADOS E DISCUSSÃO}

A tabela 3 presenta o resultado das análises metalográficas das ligas estudadas, mostrando os valores do percentual de grafita/ $\mathrm{mm}^{2}$ para cada tempo de exposição na temperatura de avaliação. O tipo de grafita predominante em todas as ligas foi a do tipo "A" e matriz 100\% perlítica. Mesmo com diferentes tempos de exposição o tipo de grafita e matriz não sofreram sensíveis variações com os ensaios.

Tabela 3. Percentual de grafita $/ \mathrm{mm}^{2}$ das ligas para os diferentes tempos de exposição à temperatura de $500^{\circ} \mathrm{C}$.

\begin{tabular}{ccc|c|c}
\hline \multicolumn{5}{c}{ \% grafita/mm } \\
\hline \multirow{2}{*}{ Liga } & \multicolumn{4}{c}{ Tempo de exposição a $\mathbf{5 0 0}{ }^{\circ} \mathbf{C}$} \\
& Oh & 1h & 2h & 4h \\
\hline C1 & 8,44 & 8,56 & 8,74 & 9,40 \\
C2 & 8,32 & 8,41 & 8,48 & 8,50 \\
C3 & 8,07 & 8,39 & 8,54 & 8,58 \\
C4 & 8,96 & 8,99 & 9,10 & 9,11 \\
\hline
\end{tabular}

Com o aumento do tempo de exposição o percentual de grafita/mm² cresce para todas as ligas. Este aumento deve-se à decomposição da cementita presente na 
matriz perlítica, a qual não possui estabilidade termodinâmica, e por difusão transforma-se em ferrita e grafita quando exposta a elevadas temperaturas. $O$ carbono proveniente da decomposição migra para as lamelas de grafita já existentes, como se pode observar no comparativo entre as micrografias em diferentes tempos de exposição da figura 3.
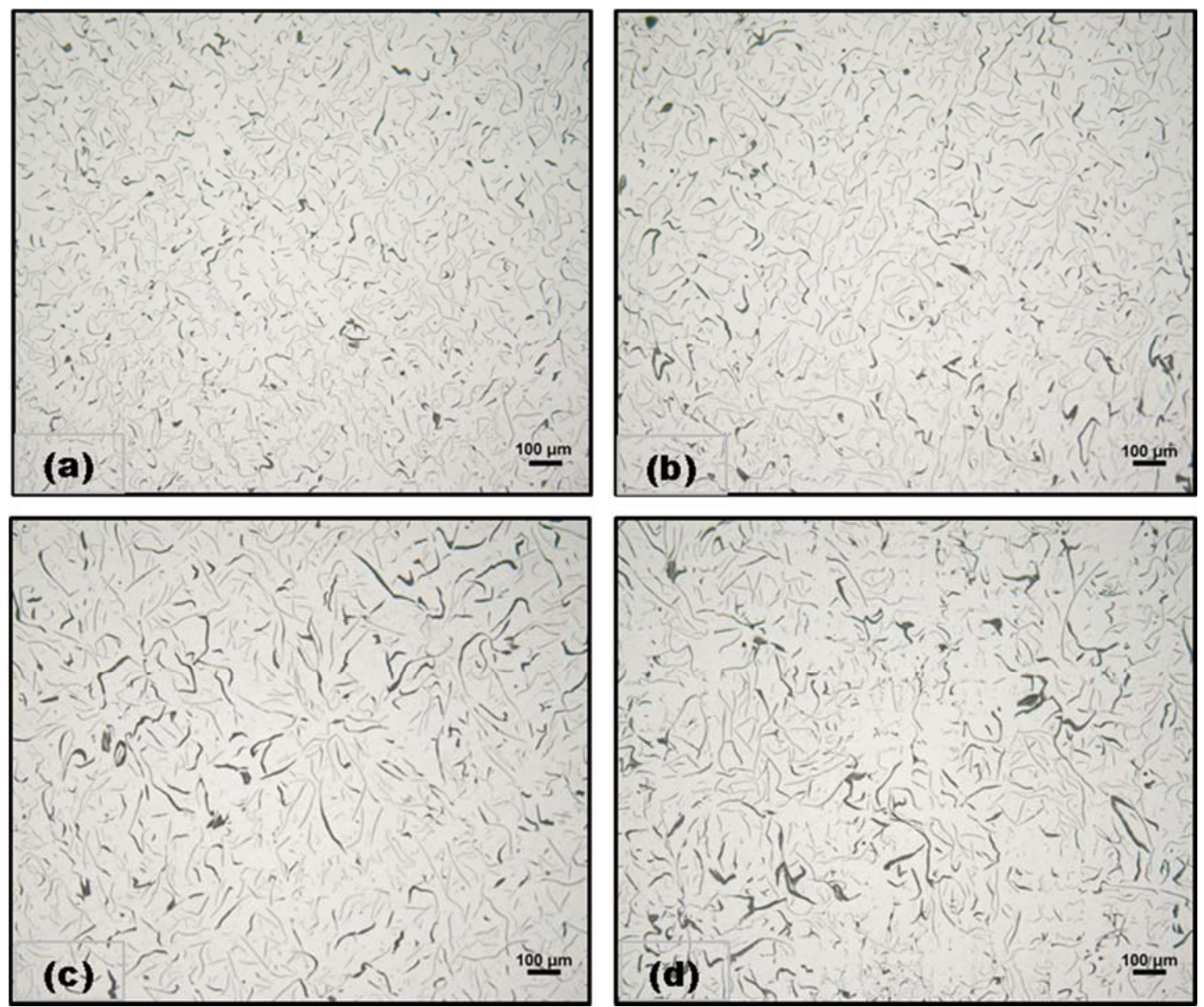

Figura 3. Grafita na liga C1 após banho a $500^{\circ} \mathrm{C}$ por (a) 0h, (b) 1h, (c) $2 \mathrm{~h}$ e (d) 4h.

A influência do tempo de exposição à elevadas temperaturas nos valores do percentual de grafita/mm² pode ser observada na figura 4. A diferença entre os valores do percentual de grafita entre as ligas deve-se aos diferentes teores dos elementos de liga $\mathrm{Cr}$ e Mo, evidenciando a influência que a presença destes elementos causa na estabilidade da microestrutura quando exposta a elevadas temperaturas.

A liga $\mathrm{C} 1$, a qual não possui níveis significativos destes elementos de liga, apresentou o maior aumento do percentual de grafita, quando comparada com as outras ligas que possuem maiores teores de $\mathrm{Cr}$ e/ou Mo. A liga C4, com elevados teores de $\mathrm{Cr}$ e Mo foi a que teve uma menor decomposição da cementita da perlita. Verifica-se, também, que a liga com alto cromo (C2) possui um efeito mais acentuado na diminuição da decomposição que a com alto molibdênio (C3).

Análises metalográficas da matriz perlítica também demostram a decomposição da cementita com o aumento do tempo de exposição e elevada temperatura. As figuras 5, 6, 7 e 8 apresentam imagens das ligas estudadas, a diferentes tempos de exposição a $500^{\circ} \mathrm{C}$. Destaca-se nas imagens regiões onde se verifica a degradação 
e esferoidização da cementita da perlita, principalmente para o ferro fundido não ligado, C1.

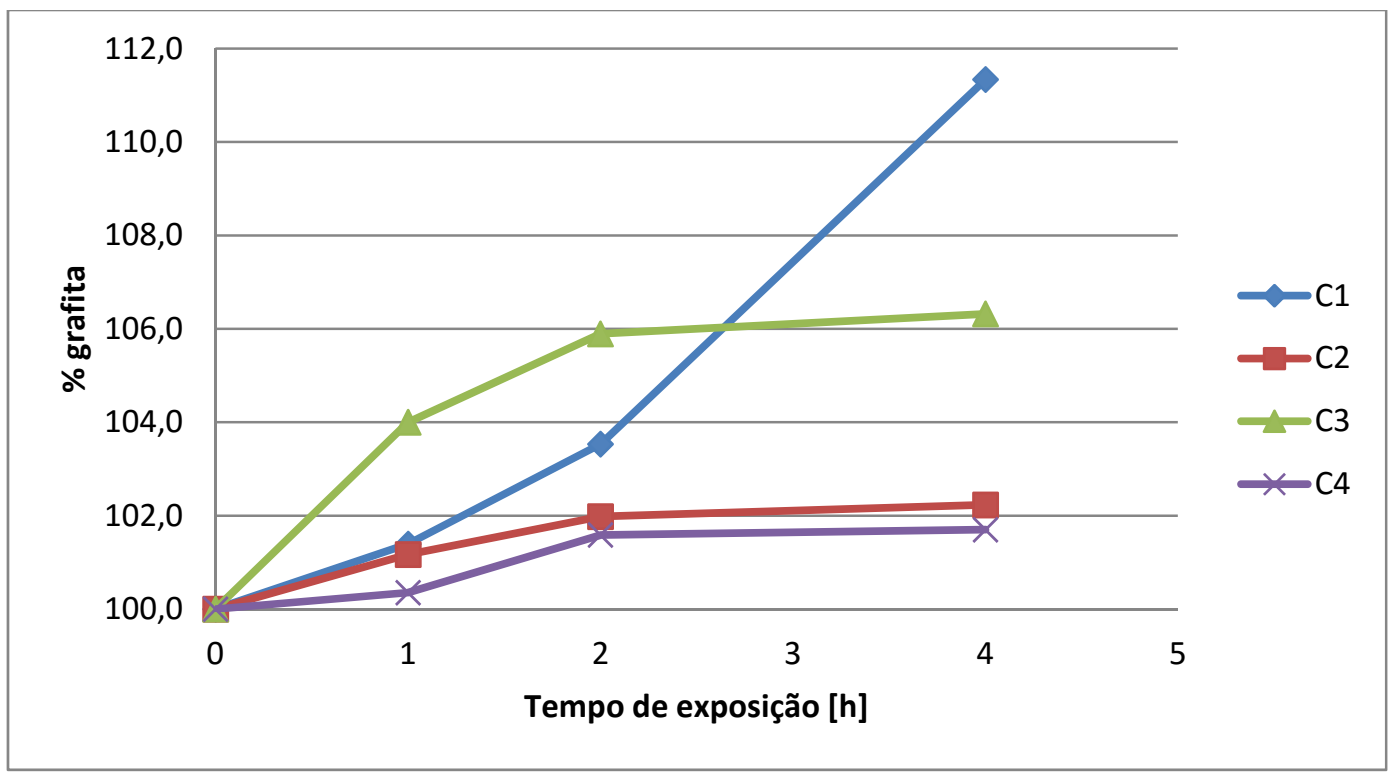

Figura 4. Percentuais de grafita/mm² tendo como parâmetro o valor à Oh.
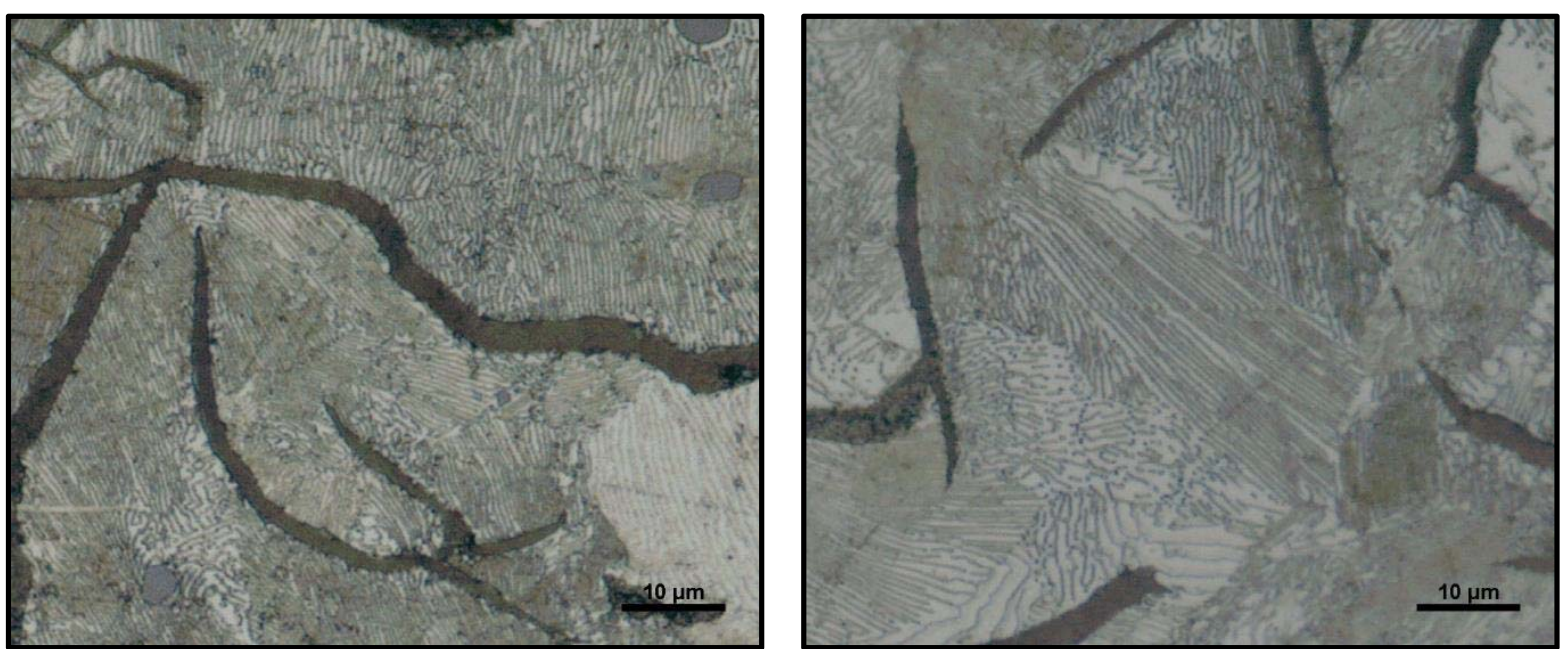

Figura 5. Matriz perlítica da liga $\mathrm{C} 1$ a $500^{\circ} \mathrm{C}$ por (a) $0 \mathrm{~h}$ e (b) $4 \mathrm{~h}$.
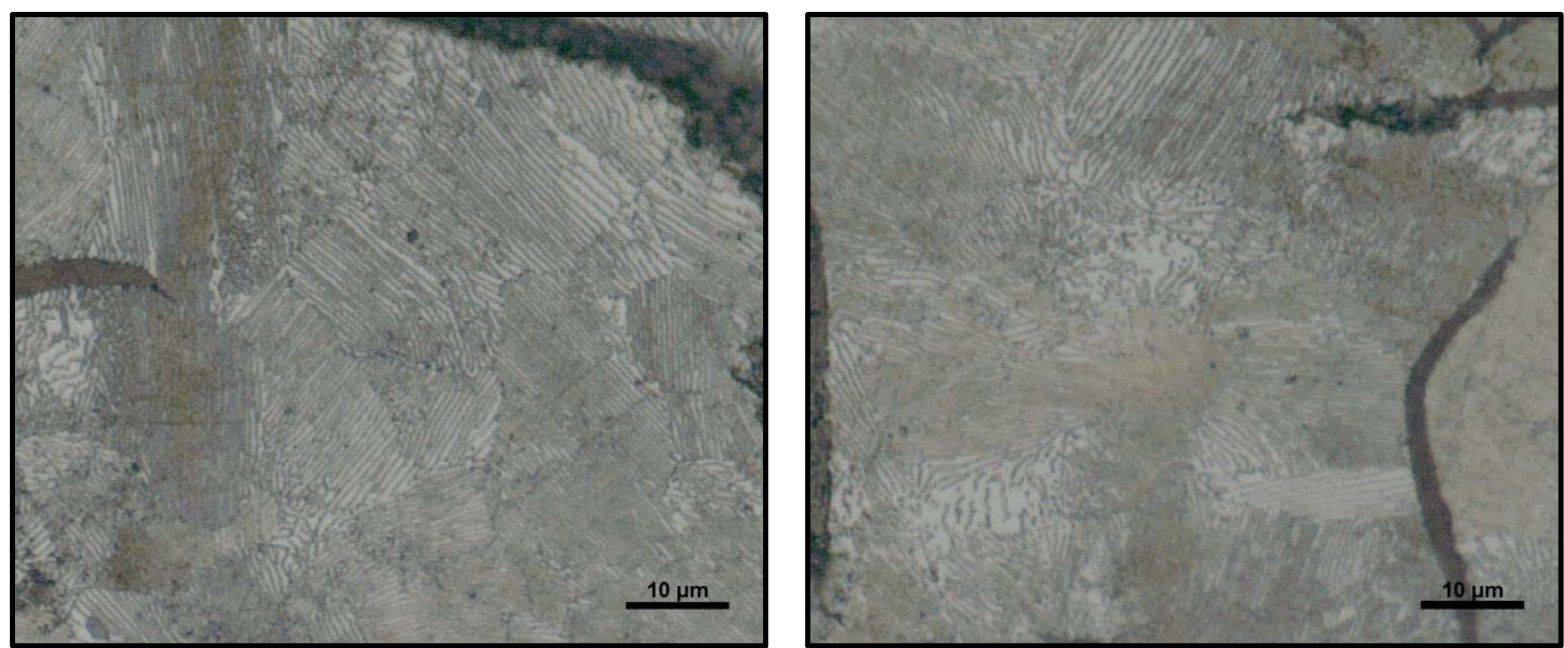

Figura 6. Matriz perlítica da liga $\mathrm{C} 2$ a $500^{\circ} \mathrm{C}$ por (a) $0 \mathrm{~h}$ e (b) $4 \mathrm{~h}$. 

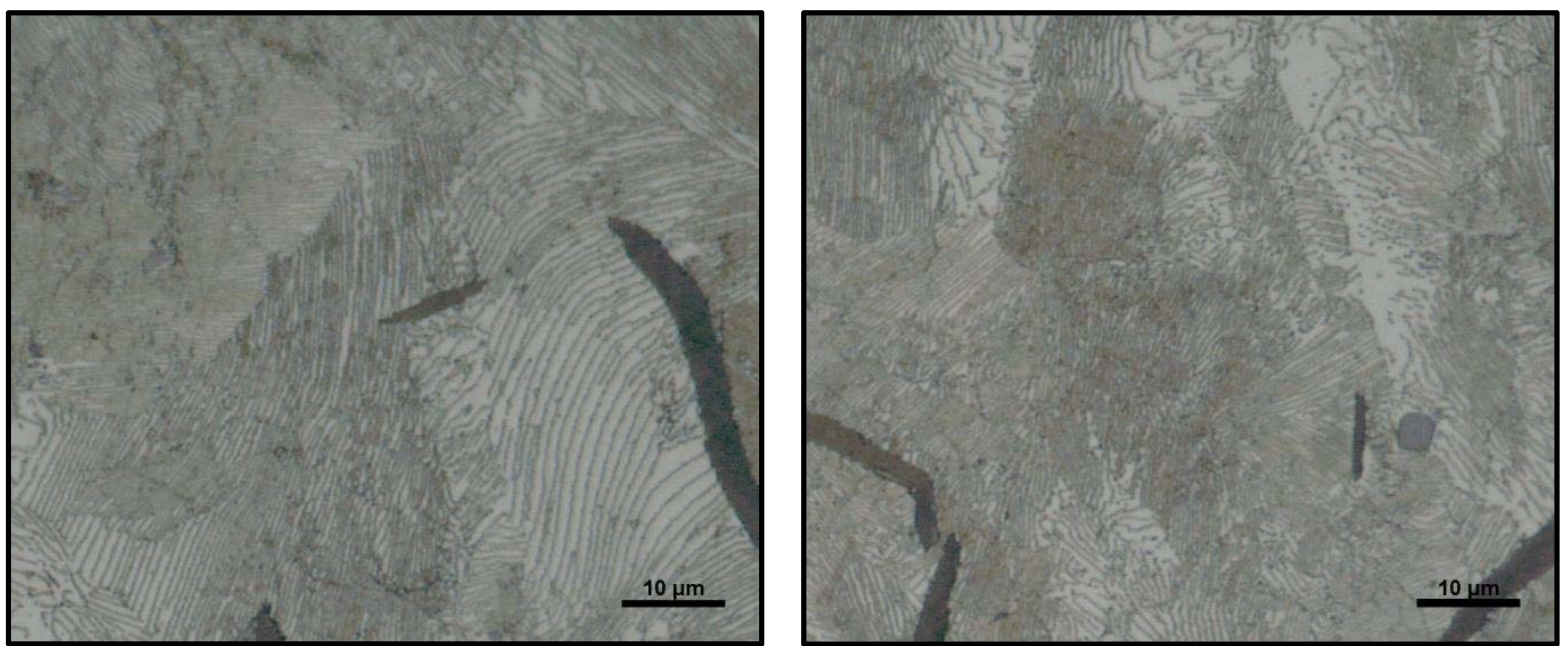

Figura 7. Matriz perlítica da liga $\mathrm{C} 3$ a $500^{\circ} \mathrm{C}$ por (a) $0 \mathrm{~h}$ e (b) $4 \mathrm{~h}$.
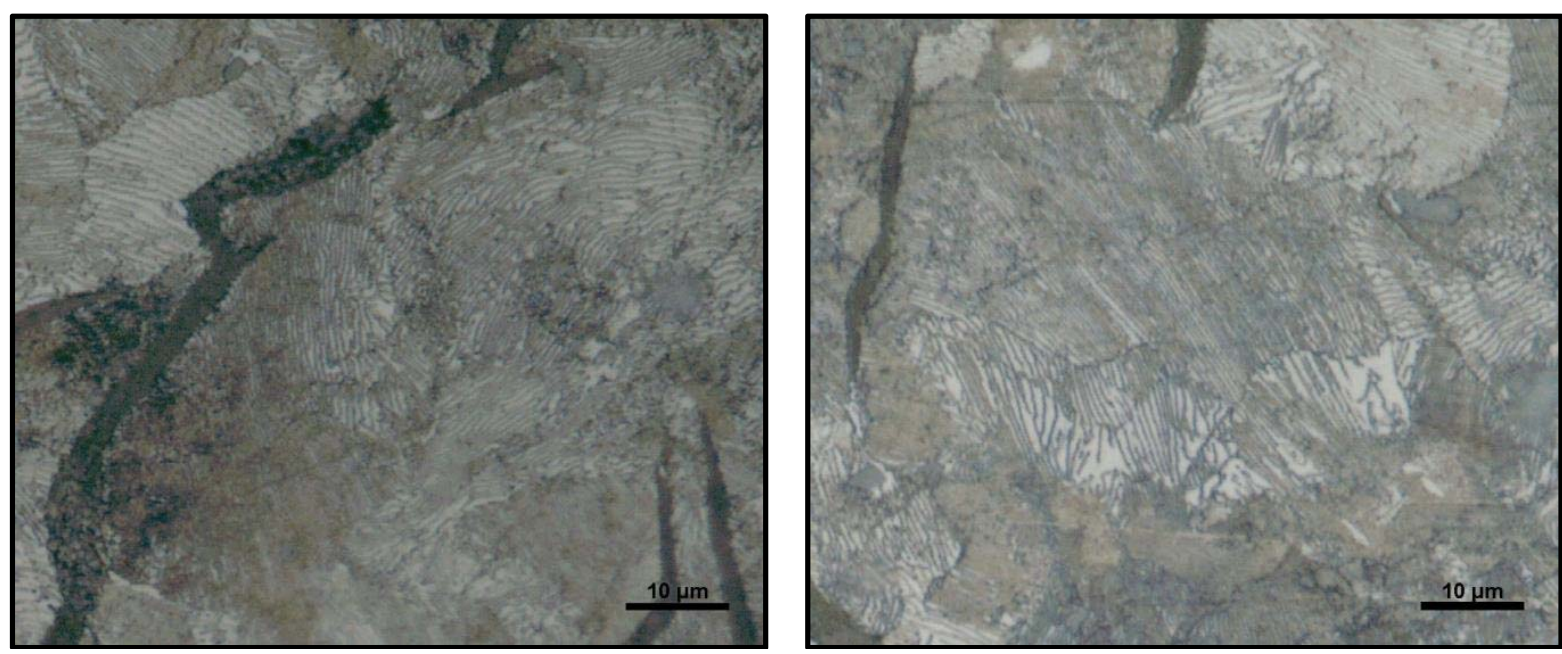

Figura 8. Matriz perlítica da liga $\mathrm{C} 4$ a $500^{\circ} \mathrm{C}$ por (a) $0 \mathrm{~h}$ e (b) $4 \mathrm{~h}$.

Adicionalmente, a tabela 4 apresenta os valores da microdureza dos corpos de prova ensaiados a diferentes tempos de exposição a $500^{\circ} \mathrm{C}$. A figura 9 expõe a variação nos percentuais de microdureza, onde se observa uma diminuição da dureza da matriz devido à degradação da perlita exposta a elevada temperatura para todas as ligas. A maior queda na microdureza ocorre no ferro fundido C1 por possuir baixos teores de elementos de liga.

Tabela 4. Microdureza para os diferentes tempos de exposição à temperatura de $500^{\circ} \mathrm{C}$.

\begin{tabular}{ccccc}
\hline \multicolumn{5}{c}{ Microdureza (HRC) } \\
\hline \multirow{2}{*}{ Liga } & \multicolumn{4}{c}{ Tempo de exposição a $\mathbf{5 0 0}^{\circ} \mathbf{C}$} \\
& 0h & 1h & 2h & 4h \\
\hline C1 & 30,6 & 30,2 & 29,4 & 28,1 \\
C2 & 32,0 & 31,5 & 31,0 & 30,4 \\
C3 & 31,5 & 31,3 & 30,8 & 30,2 \\
C4 & 31,7 & 31,1 & 30,8 & 30,2 \\
\hline
\end{tabular}




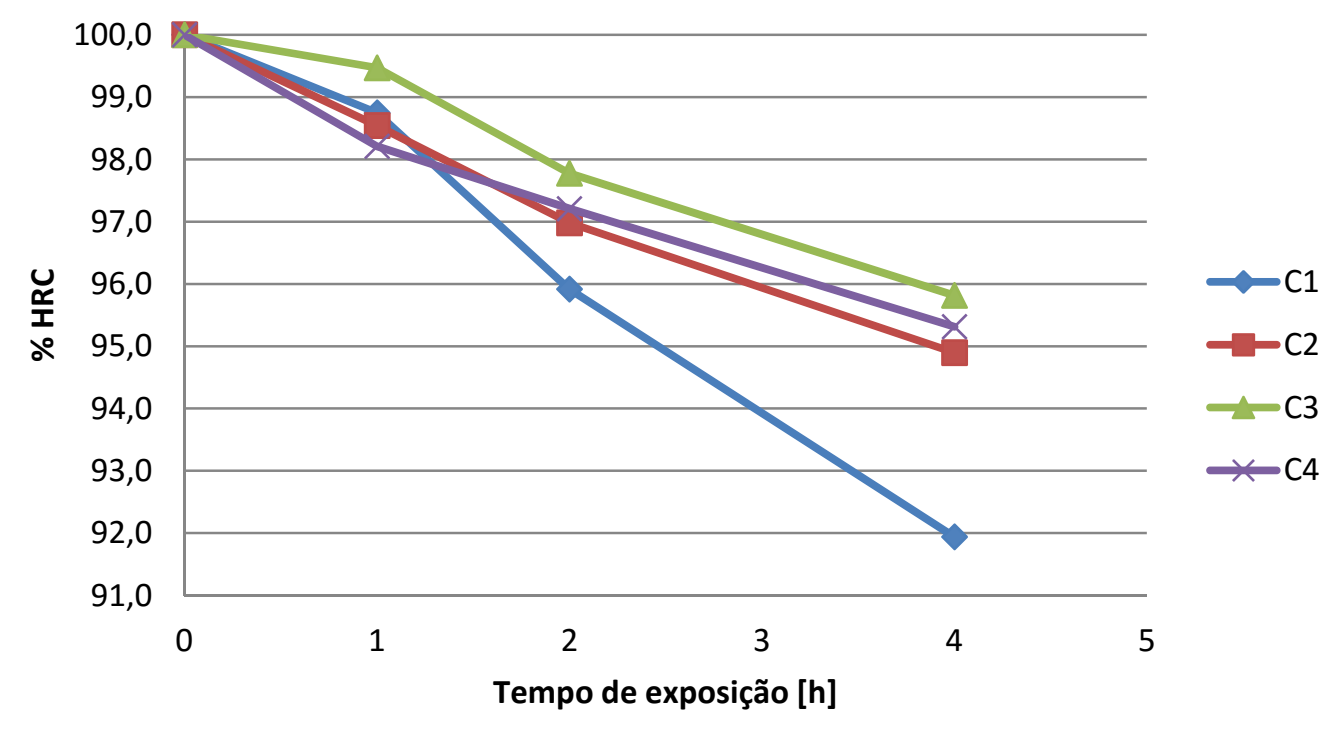

Figura 9. Valores percentuais de microdureza tendo como parâmetro os valores à $0 \mathrm{~h}$.

\section{CONCLUSÃO}

Os resultados obtidos neste estudo permitem as seguintes conclusões:

1. O processo de decomposição da perlita apresentou-se como principal causa para a degradação das propriedades do material a elevada temperatura.

2. Através das análises metalográficas evidenciou-se a influência do tempo de exposição dos materiais a altas temperaturas sobre o processo de decomposição.

3. A microdureza se mostrou sensível às alterações microestruturais, como a grafitização da cementita, podendo ser utilizada para indicar possíveis alterações na microestrutura.

4. O ferro fundido não ligado C1 apresentou menor estabilidade microestrutural do que os com adição de elementos de liga.

5. O cromo e o molibdênio foram efetivos no sentido de minimizar os efeitos da elevada temperatura sobre as propriedades mecânicas do ferro fundido cinzento.

6. O cromo mostrou um efeito mais acentuado que o molibdênio na diminuição da decomposição da perlita.

7. Aplicações a quente requerem uma análise detalhada das condições nas quais o material vai trabalhar, para a correta seleção do ferro fundido e seus elementos de liga necessários sem prejudicar seu desempenho.

\section{Agradecimentos}

À UDESC- Universidade do Estado de Santa Catarina, a Tupy S.A. e a UNISOCIESC pela disponibilidade de suas instalações e corpo técnico para a realização das atividades experimentais.

À CAPES, programa número 41002016, pela bolsa de estudos concedida. 


\section{REFERÊNCIAS}

1 Guesser WL. Propriedades Mecânicas dos Ferros Fundidos. São Paulo: Editora Blücher; 2009.

2 Nechtelberger E. The Properties of Cast Iron up to $500^{\circ} \mathrm{C}$. Stonehouse: Technicopy Ltd; 1980.

3 Samuels LE. Light Microscopy of Carbon Steels. Estados Unidos: ASM, Materials Park, 1999.

4 Palmer KB. The mechanical and physical properties of engineering grades of cast irons up to $500^{\circ} \mathrm{C}$. BCIRA Journal. 1987; November (BCIRA Report 1717):417-425.

5 Palmer KB. Design with cast irons at high temperatures - 1: growth and scaling. BCIRA Journal. 1976;(Report 1248):589-609.

6 Gilbert GNJ, White DG. Growth and scaling characteristics of flake and nodular graphite cast irons containing tin. BCIRA Journal. 1963;(11):295-318.

7 Kattus JR, Mcpherson B. Properties of Cast Iron at Elevated Temperatures. AFSM Publication; 1959. n.248.

8 Gilbert GNJ. The growth and scaling characteristics of cast irons in air steam. BCIRA Journal of Research and Development. 1959;(7):478-566.

9 Turnbull GK, Wallace JF. Molybdenum effect on gray iron at elevated temperature. AFS Transactions. 1959;(67):35-46.

10 Gundlach RB. Elevated Temperature Properties of Alloyed gray irons for diesel engine components. AFS Transactions. 1978;(86):55-64.

11 Thwaites CJ, Pryterch JC. Structural stability of flake-graphite iron alloyed with tin and chromium. Foundry Trade Journal. 1969; 115-121.

12 Black B, Burger G, Logan R; Perrin R, Gundlach R. A estrutura metalográfica e dimensional do GJS-SiMo a temperaturas elevadas. Fundição e Serviços. 2003; setembro:48-69.

13 Palmer KB. Design with cast irons at high temperatures-2: tensile, creep and rupture properties. BCIRA Journal. 1977;(25 BCIRA Report 1251):31-50.

14 Davis JR. ASM Specialty Handbook: Heat-Resistant Materials. Estados Unidos: ASM, Materials Park, 1997.

15 Bates CE. Alloy element effects on gray iron properties: part II. AFS Transactions. 1986;94:889-912. 